(C) 2017 IEEE. Personal use of this material is permitted. Permission from IEEE must be obtained for all other uses, in any current or future media, including reprinting/republishing this material for advertising or promotional purposes, creating new collective works, for resale or redistribution to servers or lists, or reuse of any copyrighted component of this work in other works. 


\title{
Statistical Analysis of Path Losses for Sectorized Wireless Networks
}

\author{
Jing Xu, Xiaojun Yan, Yuanping Zhu, Jiang Wang, Yang Yang, \\ Xiaohu Ge, Guoqiang Mao, and Olav Tirkkonen
}

\begin{abstract}
In modern mobile communication networks, such as $3 G$ and $4 G$ networks, sectorized antennas have been widely used to divide each cell into multiple sectors in order to improve coverage, spectrum efficiency and quality of service. Large-scale path loss from a transmitting antenna to a receiving antenna should include (1) propagation attenuation that depends on transmission distance, (2) shadowing that depends on surrounding environment, and (3) antenna loss that depends on a sectorized antenna pattern and transmission angle. An in-depth analysis of statistical characteristics of large-scale path losses involving with these three factors is crucial for the design, operation, evaluation and optimization of modern sectorized wireless networks. In this paper, a sectorized antenna pattern is for the first time considered in the derivation of a closed-form expression of a probability density function (PDF) of large-scale path losses. Specifically, we first discover that the normalized PDF of propagation attenuation plus shadowing, which can be approximated by the Gaussian mixture model (GMM) with all system parameters, is fully determined by our newly-defined metric $\frac{10}{\ln 10} \frac{\beta}{\sigma_{s}}$, namely the attenuation exponent $\beta$ to standard deviation of shadowing $\sigma_{s}$ ratio (ASR). The convolution of GMM and antenna loss statistics is elaborately transformed to a series of differential equations. A closed-form PDF of large-scale path losses with sectorized antenna pattern can be obtained by solving these differential equations. To reduce the computational complexity, we further prove that the exciting sources of these differential equations can be tightly approximated by weighted Gaussian functions and, thus, the final solutions (i.e. PDF of path losses) can be derived in the form of Gaussian and Dawson functions. Our analytical results are verified by extensive numerical computation and Monte Carlo simulation results, e.g. the impact of ASR on the shape of PDF of propagation attenuation plus shadowing. Compared with traditional Gaussian-fitting approach, our newlyderived PDF of large-scale path losses with sectorized antenna patterns is at least two orders of magnitude more accurate in terms of Kullback-Leibler (KL) divergence under typical
\end{abstract}

This work is partially sponsored by the National Natural Science Foundation of China under grant 61571303; the International Science and Technology Cooperation Program of China under grant 2014DFA11640; the Shanghai Natural Science Foundation under grant 15ZR1439700; the Science and Technology Commission Foundation of Shanghai under grant 15511102602.

J. Xu, X. Yan, Y. Zhu, J. Wang and Y. Yang are with the Key Laboratory of Wireless Sensor Network \& Communication, Shanghai Institute of Microsystem and Information Technology (SIMIT), Chinese Academy of Sciences (CAS), Shanghai, 200050, P. R. China. They are also with the Shanghai Research Center for Wireless Communications(WiCO), Shanghai, 201210, P. R. China. Y. Yang is the corresponding author. (Email: yang.yang@wico.sh, Tel: +86 21 60213033)

$\mathrm{X}$. Ge is with the School of Electronic Information and Communications, Huazhong University of Science and Technology, Wuhan, Hubei 430074, P. R. China. (Email: xhge@ mail.hust.edu.cn)

G. Mao is with the School of Computing and Communications, University of Technology Sydney, NSW 2007, Australia. (Email: guoqiang.mao@uts.edu.au)

O. Tirkkonen is with the Department of Communications and Networking, Aalto University, Electrical Engineering Building, Otakaari 5, 02150 Espoo, Finland. (Email: olav.tirkkonen@aalto.fi) propagation attenuation and shadowing conditions.

Index Terms-Path loss, outage probability, probability density function, Gaussian mixture model, Dawson function

\section{INTRODUCTION}

$\mathbf{I}$ N 3GPP standard TR 36.814 [1], path losses including the propagation attenuation ${ }^{1}$, shadowing and antenna pattern is one of the key metrics for the design, planning and optimization of cellular networks. Models of path losses are generally based on field measurements for given frequency ranges and particular network deployment scenarios. To obtain a probability density function (PDF) of path losses, enough path loss samples should be generated with certain configuration of the base station (BS), cell radius, deployment scenarios and specific distribution of user equipments (UEs). Simulations cannot theoretically show the impacts of channel parameters on the PDF of path losses. In the third generation (3G) cellular communication systems, to avoid the near-far [2] [3] problem, path losses are fully compensated and the received power of the desired signal at the BS from all the UEs is the same. In Long Term Evolution (LTE) and LTE-Advanced systems, the fractional power control [1] partially compensates path losses and makes a good trade-off between the cell edge user throughput and cell throughput. Therefore, the received signal to interference plus noise ratio of the 3GPP LTE uplink transmission is closely related to the path loss. In existing and future cellular networks [4] [5], antennas are often sectorized. The accurate parameterized PDF of path losses in sectorized networks can enable the tractable performance analysis [6] [7] [8] and give deep insight into the configuration of the power control and inter-cell interference mitigation [9] [10] [11] [12].

Recently, several approaches have been developed to investigate the PDF of path losses for cellular networks where BSs are equipped with omni-directional antennas. PDFs of path losses between the center of a circle and uniformly distributed UEs within the circle are derived in [13] [14]. In [14], the Kullback-Leibler (KL) divergence [15] is utilized to measure the difference between the derived PDF and the Gaussian distribution. Simulation results show that the KL divergence decreases with the increase of the standard deviation of the shadowing. In addition, the PDF of path losses is computed in a $3 \mathrm{G}$ cellular network comprised of 256 sectors. The outage probability based on the PDF of path losses for uniformly distributed UEs within the circle is investigated in [16]. For

\footnotetext{
${ }^{1}$ Propagation attenuation is also known as distance-dependent path loss
} 
Gaussianly distributed UEs in cellular networks, the closedform PDF of path losses is derived in [17] through the Taylor series expansion. Nevertheless, it is difficult to see the impact of the parameters of the BS, cell radius and deployment scenarios on the PDF of path losses. In [18], the exact analytical and approximate closed-form PDF of path losses for a hexagonal-shaped cell is derived and compared to the PDF of path losses for the circular cell. Further, the PDF of path losses for uniformly distributed UEs in the triangle-shaped cell or rhombus-shaped cell are presented in [19]. The PDF of path losses given in [20] takes the small-scale channel fading, propagation attenuation and shadowing into consideration. The PDF of the received power is derived in [21] with two mobility models when the wireless channel is assumed to have a small scale fading of Rayleigh distribution and attenuation exponent of 4. However, in the above research, the impacts of channel parameters such as attenuation exponent and standard deviation of the shadowing on the PDF of path losses are not analyzed theoretically and existing results cannot be directly utilized to derive a closed-form PDF of path losses for cellular networks where BSs are equipped with sectorized antennas.

The main obstacle to obtain the PDF of path losses in close form is the three-fold convolution related to the PDF of the propagation attenuation, shadowing and antenna pattern. In this paper, the impacts of attenuation exponent and standard deviation of shadowing on the shape of the PDF of path losses are theoretically analyzed through higher-order statistics. And the approximate PDF of path losses in close form is derived for uniformly distributed UEs in sectorized cellular networks. First, the PDF of the addition of the propagation attenuation and shadowing is elaborately parameterized by the Gaussian mixture model (GMM). The PDF of path losses can be approximately expressed as the sum of several convolutions of the Gaussian function and PDF of the antenna pattern. Then Each convolution is transformed to the solution of a differential equation. The solution of the differential equation can be approximately expressed in the form of the Dawson function [22] and Gaussian function. And the relative error of the approximate solution is bounded by the least upper bound of that of the approximation of the exciting source. With the empirical channel model, extensive numerical and simulation results not only verify the accuracy of the closed-form PDF of path losses but also show the usefulness of the closedform PDF in the design of the cell size with a given outage threshold. The main contributions of this paper are as follows:

1) We discover the normalized PDF of propagation attenuation plus shadowing is fully determined by our newlydefined metric $\frac{10}{\ln 10} \frac{\beta}{\sigma_{s}}$, namely attenuation exponent to standard deviation of shadowing ratio (ASR).

2) The PDF of propagation attenuation plus shadowing is approximated by the GMM with all system parameters.

3) We derive the closed-form PDF of large-scale path losses with sectorized antenna patterns in the form of Gaussian and Dawson functions, which is at least two orders of magnitude more accurate than traditional Gaussianfitting approach under typical propagation attenuation and shadowing conditions.
The rest of the paper is organized as follows. The system model and problem formulation are presented in Section II. In Section III, the theoretical analysis with the empirical channel model is performed to study the key metric of the PDF of the sum of the propagation attenuation and shadowing. And the GMM is utilized to parameterize the PDF of the sum of the propagation attenuation and shadowing. In Section IV, the convolution of the Gaussian function and the PDF of the antenna pattern is solved through the differential equation whose exciting source is approximated as a weighted Gaussian function. Numerical results are presented in Section V. Finally, some conclusions are made in Section VI.

\section{System Model and Problem Formulation}

\section{A. System Layout}

Without loss of generality, a three-sector circular cell layout is depicted in Fig. 1 where the polar coordinate system is used to describe node locations. The cell radius is $R$, and the cell

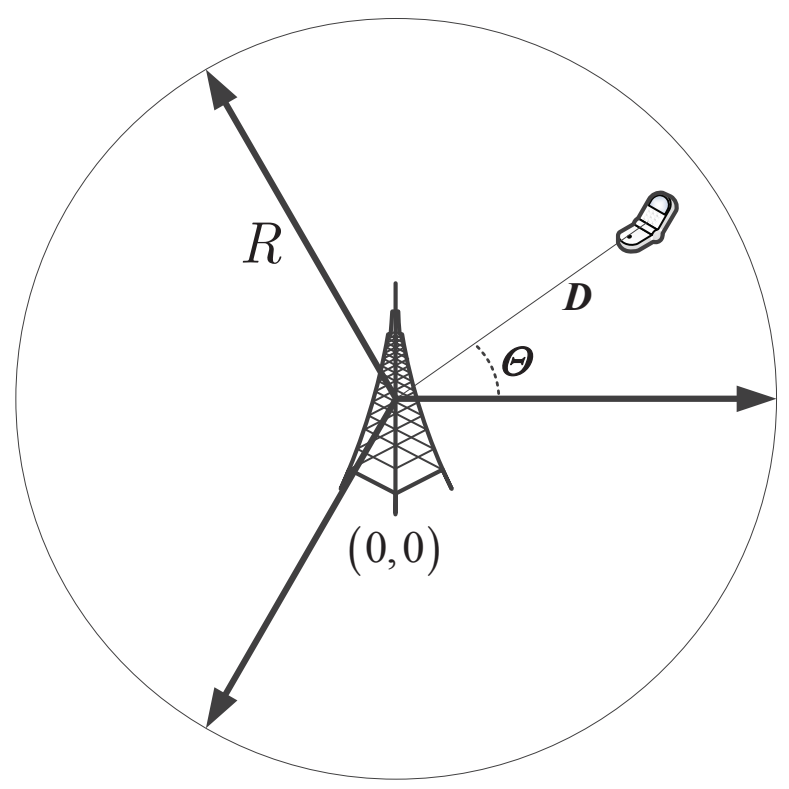

Fig. 1. Three-sector cell layout.

BS is located at the origin. The antenna boresight point in the direction of the arrows. UEs are uniformly distributed in each sector. The distance between the BS and UE is $D$, and the angle relative to the boresight pointing direction is $\Theta$. For uniformly distributed UEs, the PDFs of $\boldsymbol{D}$ and $\Theta$ are

$$
\begin{aligned}
& f_{\boldsymbol{D}}(d)=\frac{2 d}{R^{2}}, 0 \leq d \leq R, \\
& f_{\boldsymbol{\Theta}}(\theta)=\frac{k}{2 \pi},-\pi / k \leq \theta \leq \pi / k,
\end{aligned}
$$

respectively, where $k$ is the number of sectors. In Appendix A, it is proved that $\boldsymbol{D}$ and $\Theta$ are independent.

\section{B. Propagation Attenuation}

The propagation attenuation in $\mathrm{dB}$ can be expressed as

$$
\boldsymbol{L}_{1}=P L_{0}+10 \beta \log _{10} \frac{\boldsymbol{D}}{d_{0}},
$$


where $P L_{0}$ is the path loss at the reference distance $d_{0}$, and $\beta$ is the attenuation exponent.

To simplify the description, we introduce the random variable $\boldsymbol{X}=10 \beta \log _{10} \frac{\boldsymbol{D}}{d_{0}}$. $\boldsymbol{L}_{1}$ can be rewritten as

$$
\boldsymbol{L}_{1}=P L_{0}+\boldsymbol{X} \text {. }
$$

As $P L_{0}$ is a constant, studying the statistical characteristics of $\boldsymbol{L}_{1}$ is equivalent to characterizing $\boldsymbol{X}$. The PDF of the random variable $\boldsymbol{X}$ can be expressed as

$$
f_{\boldsymbol{X}}(x)=\frac{\gamma \exp (\gamma x)}{R^{2}}, x \in\left(-\infty, 10 \beta \log _{10} R\right],
$$

where

$$
\gamma=\frac{\ln 10}{5 \beta}
$$

\section{Antenna Loss}

The antenna gain $G(\theta)$ [23] at the BS can be expressed as

$$
G(\boldsymbol{\Theta})=G_{B}+A(\boldsymbol{\Theta}),
$$

where $G_{B}$ is the maximum antenna gain plus cable loss and $A(\cdot)$ is the antenna pattern. Due to the limitation of the size of UE, it is reasonable to assume that UE is equipped with the omni-directional antenna [24] [25]. The antenna pattern $A(\cdot)$ [26] [27] for the $k$-sector cell site is

$$
A(\boldsymbol{\Theta})=-\min \left(12\left(\boldsymbol{\Theta} / \theta_{k}\right)^{2}, A_{m}\right),
$$

where $\theta_{k}=\frac{7}{6} \frac{\pi}{k}$ is the $3 \mathrm{~dB}$ beam-width in degrees and $A_{m}$ is the maximum attenuation, i.e., the front-to-back ratio. Conventional values for $A_{m}$ would be $20 \mathrm{~dB}$ and $23 \mathrm{~dB}$ when the number of sectors is three and six, respectively. The antenna loss resulting from the antenna pattern is defined as

$$
\boldsymbol{Z}=\min \left(12\left(\boldsymbol{\Theta} / \theta_{k}\right)^{2}, A_{m}\right) \text {. }
$$

Because UEs are distributed in the serving cell and the angle $\theta$ is in the range $[-\pi / k, \pi / k], 12\left(\boldsymbol{\Theta} / \theta_{k}\right)^{2}$ is always less than $A_{m}$. Therefore the antenna loss $Z$ can be rewritten as

$$
\boldsymbol{Z}=12\left(\boldsymbol{\Theta} / \theta_{k}\right)^{2},-\pi / k \leq \boldsymbol{\Theta} \leq \pi / k .
$$

The PDF of $\boldsymbol{Z}$ can be expressed as

$$
f_{\boldsymbol{Z}}(z)=\frac{k \theta_{k}}{24 \pi}\left(\frac{z}{12}\right)^{-\frac{1}{2}}, z \in\left[0, \frac{12 \pi^{2}}{k^{2} \theta_{k}^{2}}\right] .
$$

\section{Path Loss}

Denote the dB-domain shadowing of the link between the BS and UE as the random variable $\boldsymbol{S}$. $\boldsymbol{S}$ can be modeled as a zero-mean, normally distributed random variable with the standard deviation $\sigma_{s} \mathrm{~dB}$.

Path losses consisting of the propagation attenuation, shadowing and antenna loss can be expressed as

$$
\boldsymbol{L}=P L_{0}-G_{B}+\boldsymbol{X}+\boldsymbol{S}+\boldsymbol{Z} .
$$

Since $\boldsymbol{X}, \boldsymbol{S}$ and $\boldsymbol{Z}$ are independent from each other [28] and the two constants $P L_{0}$ and $G_{B}$ yield a simple linear shift, the PDF of path losses $\boldsymbol{L}$ can be expressed as

$$
f_{\boldsymbol{L}}=f_{\boldsymbol{X}}(x) \otimes f_{\boldsymbol{S}}(s) \otimes f_{\boldsymbol{Z}}(z),
$$

where $\otimes$ is the convolution operation and $f_{\boldsymbol{S}}(s)$ is the PDF of $S$.

\section{ANALysis of PROPAGATION ATtENUATION Plus SHDOWING}

The sum of the propagation attenuation and shadowing can be expressed as

$$
\boldsymbol{Y}=P L_{0}-G_{B}+\boldsymbol{X}+\boldsymbol{S} .
$$

In this section, theoretical analysis based on cumulants is performed for $\boldsymbol{Y}$, and the PDF of $\boldsymbol{Y}$ is parameterized by the GMM.

\section{A. Theoretical Analysis}

We concentrate on the addition of $\boldsymbol{X}$ and $\boldsymbol{S}$. The $n$-th original moment of $\boldsymbol{X}$ can be expressed as

$$
m_{\boldsymbol{X}}^{n}=\left(10 \beta \log _{10} R\right)^{n}-\frac{n}{\gamma} m_{\boldsymbol{X}}^{n-1}, n=2,3,4, \ldots
$$

With the relationship [29] between the original moment and cumulant, the cumulants of the random variable $\boldsymbol{X}$ can be expressed as

$$
\begin{aligned}
c_{\boldsymbol{X}}^{1} & =\frac{10 \beta}{\ln 10}(\ln R-1 / 2), & c_{\boldsymbol{X}}^{2}=\left(\frac{10 \beta}{\ln 10}\right)^{2} / 4, \\
c_{\boldsymbol{X}}^{3} & =-\left(\frac{10 \beta}{\ln 10}\right)^{3} / 4, & c_{\boldsymbol{X}}^{4}=3\left(\frac{10 \beta}{\ln 10}\right)^{4} / 8, \\
c_{\boldsymbol{X}}^{5} & =-3\left(\frac{10 \beta}{\ln 10}\right)^{5} / 4, & \ldots
\end{aligned}
$$

Because $\boldsymbol{X}$ and $\boldsymbol{S}$ are independent, and the shadowing $\boldsymbol{S}$ is a zero-mean Gaussianly distributed random variable with the standard deviation $\sigma_{s} \mathrm{~dB}$, the normalized cumulants of $\boldsymbol{Y}$ are

$\tilde{c}_{\boldsymbol{X}+\boldsymbol{S}}^{1}=0, \tilde{c}_{\boldsymbol{X}+\boldsymbol{S}}^{2}=1, \tilde{c}_{\boldsymbol{X}+\boldsymbol{S}}^{3}=-\frac{1}{4} \frac{\left(\frac{10}{\ln 10} \frac{\beta}{\sigma_{s}}\right)^{3}}{\left[\left(\frac{10}{\ln 10} \frac{\beta}{\sigma_{s}}\right)^{2} / 4+1\right]^{\frac{3}{2}}}$,

$\tilde{c}_{\boldsymbol{X}+\boldsymbol{S}}^{4}=\frac{3}{8} \frac{\left(\frac{10}{\ln 10} \frac{\beta}{\sigma_{s}}\right)^{4}}{\left[\left(\frac{10}{\ln 10} \frac{\beta}{\sigma_{s}}\right)^{2} / 4+1\right]^{2}}$,

$\tilde{c}_{\boldsymbol{X}+\boldsymbol{S}}^{5}=-\frac{3}{4} \frac{\left(\frac{10}{\ln 10} \frac{\beta}{\sigma_{s}}\right)^{5}}{\left[\left(\frac{10}{\ln 10} \frac{\beta}{\sigma_{s}}\right)^{2} / 4+1\right]^{\frac{5}{2}}}, \ldots$

If the cumulants of orders higher than two asymptotically approach zero, the corresponding distribution is asymptotically normal. Therefore the metric $\frac{10}{\ln 10} \frac{\beta}{\sigma_{s}}$ defined as ASR determines the shape of the PDF of $\boldsymbol{Y}$. When the ASR is small, the PDF can be approximated as the normal distribution. Otherwise, the PDF will greatly deviate from the normal distribution. In other hand, if the ASRs are equal for different values of the attenuation exponent and standard deviation of the shadowing, the corresponding PDFs of the normalized $\boldsymbol{Y}$ are identical. From (15), we can see that the mean of $\boldsymbol{Y}$ is determined by the path loss at the reference distance $P L_{0}$, antenna gain $G_{B}$, cell radius $R$ and attenuation exponent $\beta$. The variance of $\boldsymbol{Y}$ only depends on the attenuation exponent $\beta$ and standard deviation of the shadowing $\sigma_{s}$. 


\section{B. Gaussian Mixture Model}

With the results derived in [13] [14], the PDF of $\boldsymbol{Y}$ can be expressed as

$$
f_{\boldsymbol{Y}}(y)=c_{0} \exp \left(q_{0} y_{0}\right) \operatorname{erfc}\left(y_{0}\right),
$$

where

$$
\begin{aligned}
c_{0}= & \frac{\ln 10}{10 \beta R^{2}} \exp \left[\frac{2 \sigma_{s}^{2}(\ln 10)^{2}-20 P L_{0} \beta \ln 10}{(10 \beta)^{2}}\right] \\
& \times \exp \left(\frac{b_{0} \ln 10}{5 \beta}\right), \\
q_{0}= & \frac{\sqrt{2} \ln 10}{5} \frac{\sigma_{s}}{\beta}, y_{0}=\frac{y-b_{0}}{\sqrt{2} \sigma_{s}}, \\
b_{0}= & P L_{0}-G_{B}+10 \beta \log _{10} R-\frac{2(\ln 10) \sigma_{s}^{2}}{10 \beta} .
\end{aligned}
$$

If $f_{\boldsymbol{Y}}(y)$ is directly approximated by the GMM, the impact of network parameters on the PDF of $\boldsymbol{Y}$ cannot be shown explicitly. On the other hand, as the limit of erfc $(x)$ as $x$ approaches negative infinity is 2 , the function $\operatorname{erfc}(\cdot)$ cannot be approximated by the GMM. To parameterize $f_{\boldsymbol{Y}}(y)$ with the GMM, (17) is rewritten as

$$
f_{\boldsymbol{Y}}(y)=c_{0} \frac{\exp \left(q_{0} y_{0}\right)}{p\left(y_{0}\right)} g\left(y_{0}\right),
$$

where

$$
g\left(y_{0}\right)=p\left(y_{0}\right) \operatorname{erfc}\left(y_{0}\right) .
$$

If the limit of $g\left(y_{0}\right)$ as $y_{0}$ approaches infinity is $0, g\left(y_{0}\right)$ may be approximated by the GMM. To simplify the derivation, $p\left(y_{0}\right)$ is chosen as $\exp \left(y_{0}\right)$ to satisfy the above condition. The function $g\left(y_{0}\right)$ can then be expressed as

$$
g\left(y_{0}\right)=\exp \left(y_{0}\right) \operatorname{erfc}\left(y_{0}\right) .
$$

Considering $g\left(y_{0}\right)$ as a function of $y_{0}$ and all the network parameters are included in the intermediate variable $y_{0}, g\left(y_{0}\right)$ is independent of network parameters. Furthermore, $g\left(y_{0}\right)$ can be tightly approximated by the $M$-order GMM as

$$
g\left(y_{0}\right) \approx g_{M}\left(y_{0}\right)=\sum_{i=1}^{M} a_{i} \exp \left[-\frac{\left(y_{0}-u_{i}\right)^{2}}{\left(2 \sigma_{i}^{2}\right)}\right] .
$$

Since $g\left(y_{0}\right)$ is independent of network parameters, the performance of $g_{M}\left(y_{0}\right)$ in terms of $y_{0}$ is also independent of network parameters which are all retained in $c_{0} \exp \left(\left(q_{0}-1\right) y_{0}\right)$. Using the trust region algorithm [30] [31], the parameters $a_{i}$, $u_{i}$ and $\sigma_{i}$ for $M=2,4,6,8$ can be found, and the results are listed in Table I. The performance of the GMM approximation is depicted in Fig. 2. After the normalization of $g_{M}\left(y_{0}\right)$ and $g\left(y_{0}\right)$, the KL divergence can be used to the measure the performance of the GMM approximation. The KL divergence of the GMM approximation from $g\left(y_{0}\right)$ is presented in Table II. It can be seen that when the order of the GMM is not less than four, the GMM can provide satisfactory performance.

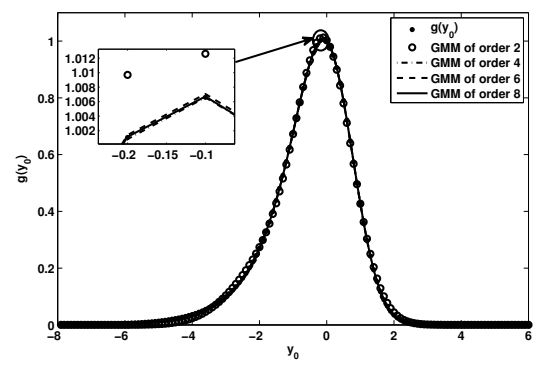

Fig. 2. $g\left(y_{0}\right)$ and its GMM approximation.

Substituting (22) into (19), the PDF of $\boldsymbol{Y}$ can be rewritten as

$$
\begin{aligned}
f_{\boldsymbol{Y}}(y) & \approx f_{\boldsymbol{Y}, M}(y) \\
& =c_{0} \sum_{i=1}^{M} \tilde{a}_{i} \sqrt{2 \pi \tilde{\sigma}_{i}^{2}} \frac{1}{\sqrt{2 \pi \tilde{\sigma}_{i}^{2}}} \exp \left[-\frac{\left(y-\tilde{u}_{i}\right)^{2}}{\left(2 \tilde{\sigma}_{i}^{2}\right)}\right],
\end{aligned}
$$

\begin{tabular}{|c|c|c|c|}
\hline Order & $a_{i}$ & $u_{i}$ & $\overline{\sigma_{i}}$ \\
\hline \multirow[t]{2}{*}{$\mathrm{M}=2$} & 0.8397 & -0.02036 & $1.139 / \sqrt{2}$ \\
\hline & 0.2782 & -1.268 & $1.737 / \sqrt{2}$ \\
\hline \multirow{4}{*}{$\mathrm{M}=4$} & 0.6377 & 0.1862 & $1.039 / \sqrt{2}$ \\
\hline & 0.3969 & -0.568 & $1.182 / \sqrt{2}$ \\
\hline & 0.02941 & -2.889 & $2.319 / \sqrt{2}$ \\
\hline & 0.1593 & -1.522 & $1.558 / \sqrt{2}$ \\
\hline \multirow{6}{*}{$M=6$} & 0.7937 & 0.1268 & $1.06 / \sqrt{2}$ \\
\hline & 0.232 & -0.7288 & $1.059 / \sqrt{2}$ \\
\hline & 0.1749 & -1.25 & $1.275 / \sqrt{2}$ \\
\hline & 0.06415 & -2.217 & $1.423 / \sqrt{2}$ \\
\hline & 0.02033 & -3.277 & $1.796 / \sqrt{2}$ \\
\hline & 0.004299 & -4.304 & $2.691 / \sqrt{2}$ \\
\hline \multirow{8}{*}{$\mathrm{M}=8$} & 0.8758 & -0.04354 & $1.09 / \sqrt{2}$ \\
\hline & 0.2134 & -1.089 & $0.9507 / \sqrt{2}$ \\
\hline & 0.09447 & 0.5655 & $0.9247 / \sqrt{2}$ \\
\hline & 0.1284 & -1.931 & $0.9949 / \sqrt{2}$ \\
\hline & 0.03894 & -2.911 & $1.018 / \sqrt{2}$ \\
\hline & 0.01358 & -3.743 & $1.224 / \sqrt{2}$ \\
\hline & 0.007431 & -4.163 & $1.855 / \sqrt{2}$ \\
\hline & 0.002163 & -4.537 & $3.005 / \sqrt{2}$ \\
\hline
\end{tabular}

where

$$
\begin{aligned}
& \tilde{a}_{i}=a_{i} \exp \left\{\left[\left(u_{i}+\frac{q_{0}-1}{2} 2 \sigma_{i}^{2}\right)^{2}-u_{i}^{2}\right] /\left(2 \sigma_{i}^{2}\right)\right\}, \\
& \tilde{u}_{i}=b_{0}+\sqrt{2} \sigma_{s}\left(u_{i}+\frac{q_{0}-1}{2} 2 \sigma_{i}^{2}\right), \tilde{\sigma}_{i}=\sqrt{2} \sigma_{s} \sigma_{i} .
\end{aligned}
$$

To be a PDF, the approximated function $f_{\boldsymbol{Y}, M}(y)$ needs to be

TABLE I

PARAMETERS OF GMM 
TABLE II

KL DIVERGENCE OF GMM APPROXIMATION

\begin{tabular}{cc}
\hline Order of GMM & KL divergence \\
\hline$M=2$ & $1.85 \times 10^{-2}$ \\
\hline$M=4$ & $4.98 \times 10^{-4}$ \\
\hline$M=6$ & $3.93 \times 10^{-5}$ \\
\hline$M=8$ & $7.76 \times 10^{-6}$ \\
\hline
\end{tabular}

normalized as

$$
\hat{f}_{\boldsymbol{Y}, M}(y)=\sum_{i=1}^{M} \lambda_{i} N\left(y ; \tilde{u}_{i}, \tilde{\sigma}_{i}^{2}\right),
$$

where

$$
\begin{aligned}
& \lambda_{i}=\frac{\tilde{a}_{i} \sqrt{2 \pi \tilde{\sigma}_{i}^{2}}}{\sum_{i=1}^{M} \tilde{a}_{i} \sqrt{2 \pi \tilde{\sigma}_{i}^{2}}} \\
& N\left(y ; \tilde{u}_{i}, \tilde{\sigma}_{i}^{2}\right)=\frac{1}{\sqrt{2 \pi \tilde{\sigma}_{i}^{2}}} \exp \left[-\left(y-\tilde{u}_{i}\right)^{2} /\left(2 \tilde{\sigma}_{i}^{2}\right)\right] .
\end{aligned}
$$

Numerical results in Section V show that the GMM is more accurate than the Gaussian approximation. In the procedure to get the GMM, we aim at $\sigma_{i}$ as big as possible. The reason will be given in sub-section B of Section IV.

\section{Probability Density Function of Path Loss with Sectorized Antenna Pattern}

In this section, the approximate PDF of path losses $\boldsymbol{L}$ consisting of the propagation attenuation, shadowing and antenna loss is derived.

\section{A. Convolution of Gaussian Function and PDF of Antenna Loss}

The PDF of path losses is the convolution of the PDF of $\boldsymbol{Y}$ and $Z$

$$
f_{\boldsymbol{L}}(l)=\int_{0}^{A_{L}} f_{\boldsymbol{Z}}(z) f_{\boldsymbol{Y}}(l-z) d z
$$

where

$$
A_{L}=\frac{12 \pi^{2}}{k^{2} \theta_{k}^{2}} .
$$

Substituting (25) into (27), the PDF of path losses can be approximated as

$$
\tilde{f}_{\boldsymbol{L}}(l)=\sum_{i=1}^{M} \lambda_{i} f_{\boldsymbol{L}, i}(l),
$$

where

$$
f_{\boldsymbol{L}, i}(l)=\int_{0}^{A_{L}} f_{\boldsymbol{Z}}(z) N\left(l-z ; \tilde{u}_{i}, \tilde{\sigma}_{i}^{2}\right) d z .
$$

Therefore the function $\tilde{f}_{\boldsymbol{L}}(l)$ is the weighted sum of convolutions of the PDF of the antenna loss and Gaussian function. Differentiating (30), we get

$$
f_{\boldsymbol{L}, i}^{\prime}(l)+P_{i}(l) f_{\boldsymbol{L}, i}(l)=Q_{i}(l),
$$

where

$$
\begin{aligned}
Q_{i}(l) & =\frac{Q_{0}}{\tilde{\sigma}_{i}^{2}} \int_{0}^{A_{L}} \frac{3\left(\sqrt{A_{L}}\right)^{-3} \sqrt{z}}{2 \sqrt{2 \pi \tilde{\sigma}_{i}^{2}}} \exp \left[-\frac{\left(l-z-\tilde{u}_{i}\right)^{2}}{2 \tilde{\sigma}_{i}^{2}}\right] d z, \\
P_{i}(l) & =\frac{l-\tilde{u}_{i}}{\tilde{\sigma}_{i}^{2}}, \\
Q_{0} & =\frac{1}{3 \sqrt{12} \tilde{\sigma}_{i}^{2}} A_{L}^{2} .
\end{aligned}
$$

The general solution of the above differential equation is

$$
\begin{aligned}
& f_{\boldsymbol{L}, i}(l)=\exp \left(-\int_{l_{0}}^{l} P_{i}(s) d s\right) \\
& \times\left[\int_{l_{0}}^{l} Q_{i}(t) \exp \left(\int_{l_{0}}^{t} P_{i}(s) d s\right) d t+f_{\boldsymbol{L}, i}\left(l_{0}\right)\right] .
\end{aligned}
$$

The detailed proof is given in Appendix B. Since the integral of $P_{i}(l)$ is known, the obstacle to achieve a closed-form expression for (33) is the integral of $Q_{i}(l)$. From (32a), the normalized $Q_{i}(l)$ can be considered as the PDF of the random variable $\boldsymbol{Z}_{3, i}=\boldsymbol{Z}_{1}+\boldsymbol{Z}_{2, i}$ where $\boldsymbol{Z}_{1}$ has the PDF of $\frac{3}{2}\left(\sqrt{A_{L}}\right)^{-3} \sqrt{z_{1}}, z_{1} \in\left[0, A_{L}\right]$, and $Z_{2, i}$ is the Gaussian random variable with the mean $\tilde{u}_{i}$ and variance $\tilde{\sigma}_{i}^{2}$. In the next sub-section, we will prove that the PDF of $\boldsymbol{Z}_{3, i}$ can be well approximated as a Gaussian function.

\section{B. Gaussian Approximation of Exciting Source}

The $n$-th order original moment of $\boldsymbol{Z}_{1}$ can be expressed as

$$
m_{\boldsymbol{Z}_{1}}^{n}=\frac{3}{2 n+3} A_{L}^{n} .
$$

The cumulants of the Gaussian random variable $\boldsymbol{Z}_{2, i}$ can be written as

$$
c_{\boldsymbol{Z}_{2, i}}^{1}=\tilde{u}_{i}, c_{\boldsymbol{Z}_{2, i}}^{2}=\tilde{\sigma}_{i}^{2}, c_{\boldsymbol{Z}_{2, i}}^{n}=0, n>2 .
$$

Because $\boldsymbol{Z}_{1}$ and $\boldsymbol{Z}_{2, i}$ are independent, the cumulants of $\boldsymbol{Z}_{3, i}$ are

$$
\begin{array}{rlrl}
c_{\boldsymbol{Z}_{3, i}}^{1} & =\frac{2}{5} A_{L}+\tilde{u}_{i}, & c_{\boldsymbol{Z}_{3, i}}^{2} & =\frac{12}{175} A_{L}^{2}+\tilde{\sigma}_{i}^{2}, \\
c_{\boldsymbol{Z}_{3, i}}^{3} & =-\frac{16}{2625} A_{L}^{3}, & c_{\boldsymbol{Z}_{3, i}}^{4} & =-\frac{1504}{336875} A_{L}^{4}, \\
c_{\boldsymbol{Z}_{3, i}}^{5} & =\frac{45824}{21896875} A_{L}^{5}, & \ldots
\end{array}
$$

The cumulants of the normalized random variable $\bar{Z}_{3, i}=$ $\frac{\boldsymbol{Z}_{3, i}-c_{\boldsymbol{Z}_{3, i}}^{1}}{\sqrt{c_{\boldsymbol{Z}_{3, i}}^{2}}}$ can be expressed as

$$
\begin{aligned}
c_{\bar{Z}_{3, i}}^{1} & =0, c_{\bar{Z}_{3, i}}^{2}=1, c_{\bar{Z}_{3, i}}^{3}=-\frac{\frac{16}{2625}}{\left(\frac{12}{175}+\tilde{\sigma}_{i}^{2} / A_{L}^{2}\right)^{\frac{3}{2}}}, \\
c_{\bar{Z}_{3, i}}^{4} & =-\frac{\frac{1504}{336875}}{\left(\frac{12}{175}+\tilde{\sigma}_{i}^{2} / A_{L}^{2}\right)^{2}}, \\
c_{\bar{Z}_{3, i}}^{5} & =\frac{\frac{45824}{21896875}}{\left(\frac{12}{175}+\tilde{\sigma}_{i}^{2} / A_{L}^{2}\right)^{\frac{5}{2}}}, \ldots
\end{aligned}
$$

We define the standard deviation to the maximum antenna loss ratio (DLR) as $\tilde{\sigma}_{i} / A_{L}$. Equation (37) shows that the limit 
of the $n$-th order cumulant of $\overline{\boldsymbol{Z}}_{3, i}(n=3,4,5, \ldots)$ as DLR approaches infinity is zero. When the $3 \mathrm{~dB}$ beam-width $\theta_{k}$ is $\frac{7}{6} \frac{\pi}{k}$ as specified in [1] [27] and $\tilde{\sigma}_{i}$ is $4 \mathrm{~dB}$, the cumulants of $Z_{3, i}$ are

$$
\begin{aligned}
& c_{\overline{\boldsymbol{Z}}_{3, i}}^{1}=0, c_{\overline{\boldsymbol{Z}}_{3, i}}^{2}=1, c_{\overline{\boldsymbol{Z}}_{3, i}}^{3} \approx-0.0424, \\
& c_{\overline{\boldsymbol{Z}}_{3, i}}^{4} \approx-0.059, c_{\overline{\boldsymbol{Z}}_{3, i}}^{5} \approx 0.053, \ldots
\end{aligned}
$$

From (38), we can see that $\boldsymbol{Z}_{3, i}$ can be well approximated as the Gaussian random variable with the mean $m_{Z_{3, i}}^{1}=\frac{2}{5} A_{L}+$ $\tilde{u}_{i}$ and variance $c_{\boldsymbol{Z}_{3, i}}^{2}=\frac{12}{175} A_{L}^{2}+\tilde{\sigma}_{i}^{2}$. The requirement to make $\sigma_{i}$ as big as possible (as stated in sub-section A of Section III) will result in the cumulants of $c_{\bar{Z}_{3, i}}^{n}$ of orders higher than two approaching zero quickly. Therefore, when $\tilde{\sigma}_{i}$ is big enough, the function $Q_{i}(l)$ defined in (32a) can be well approximated as

$$
\hat{Q}_{i}(l)=\frac{Q_{0}}{\tilde{\sigma}_{i}^{2}} \frac{1}{\sqrt{2 \pi c_{\boldsymbol{Z}_{3, i}}^{2}}} \exp \left[-\frac{\left(l-m_{\boldsymbol{Z}_{3, i}}^{1}\right)^{2}}{2 c_{\boldsymbol{Z}_{3, i}}^{2}}\right] .
$$

Numerical results in Section V show that this approximation is accurate, especially when $\tilde{\sigma}_{i}$ is large.

\section{Differential Equation with Approximated Exciting Source}

With the approximated exciting source $\hat{Q}_{i}(l),(31)$ can be rewritten as

$$
\hat{f}_{\boldsymbol{L}, i}^{\prime}(l)+P_{i}(l) \hat{f}_{\boldsymbol{L}, i}(l)=\hat{Q}_{i}(l),
$$

where $\hat{f}_{\boldsymbol{L}, i}(l)$ is the solution that can be written as

$$
\begin{aligned}
& \hat{f}_{\boldsymbol{L}, i}(l)=\left(\exp \left(-\int_{l_{0}}^{l} P_{i}(s) d s\right)\right) \\
& \quad \times\left[\int_{l_{0}}^{l} \hat{Q}_{i}(t) \exp \left(\int_{l_{0}}^{t} P_{i}(s) d s\right) d t+\hat{f}_{\boldsymbol{L}, i}\left(l_{0}\right)\right] .
\end{aligned}
$$

Replacing $\hat{f}_{\boldsymbol{L}, i}\left(l_{0}\right)$ by $f_{\boldsymbol{L}, i}\left(l_{0}\right)$ which can be achieved through a numerical computation, (41) can be rewritten as

$$
\begin{aligned}
& \hat{f}_{\boldsymbol{L}, i}(l)=\left(\exp \left(-\int_{l_{0}}^{l} P_{i}(s) d s\right)\right) \\
& \quad \times\left[\int_{l_{0}}^{l} \hat{Q}_{i}(t) \exp \left(\int_{l_{0}}^{t} P_{i}(s) d s\right) d t+f_{\boldsymbol{L}, i}\left(l_{0}\right)\right] .
\end{aligned}
$$

To get a succinct expression and have deep insight into the PDF of path losses, we assume

$$
\hat{f}_{\boldsymbol{L}, i}(l)=G_{i}(l) \hat{Q}_{i}(l) .
$$

Substituting (43) into (40), the differential equation can be rewritten as

$$
G_{i}^{\prime}(l)+\frac{l-u_{g, i}}{\tilde{\sigma}_{g, i}^{2}} G_{i}(l)=1,
$$

where

$$
u_{g, i}=\frac{c_{\boldsymbol{Z}_{3, i}}^{2} \tilde{u}_{i}-\tilde{\sigma}_{i}^{2} m_{\boldsymbol{Z}_{3, i}}^{1}}{c_{\boldsymbol{Z}_{3, i}}^{2}-\tilde{\sigma}_{i}^{2}}, \tilde{\sigma}_{g, i}^{2}=\frac{\tilde{\sigma}_{i}^{2} c_{\boldsymbol{Z}_{3, i}}^{2}}{c_{\boldsymbol{Z}_{3, i}}^{2}-\tilde{\sigma}_{i}^{2}} .
$$

Setting $l_{0}=u_{g, i}$, the solution of (44) can be expressed as

$$
\begin{aligned}
G_{i}(l)= & \sqrt{2 \tilde{\sigma}_{g, i}^{2}} D_{+}\left(\frac{l-u_{g, i}}{\sqrt{2 \tilde{\sigma}_{g, i}^{2}}}\right) \\
& +\exp \left[-\frac{\left(l-u_{g, i}\right)^{2}}{2 \tilde{\sigma}_{g, i}^{2}}\right] G_{i}\left(u_{g, i}\right),
\end{aligned}
$$

where $D_{+}(x)$ is the Dawson function that is defined as

$$
D_{+}(x)=\exp \left(-x^{2}\right) \int_{0}^{x} \exp \left(t^{2}\right) d t .
$$

Substituting (46) into (43), the function $\hat{f}_{\boldsymbol{L}, i}(l)$ can be expressed as

$$
\begin{aligned}
\hat{f}_{\boldsymbol{L}, i}(l)= & \sqrt{2 \tilde{\sigma}_{g, i}^{2}} \hat{Q}_{i}(l) D_{+}\left(\frac{l-u_{g, i}}{\sqrt{2 \tilde{\sigma}_{g, i}^{2}}}\right) \\
& +\exp \left[-\frac{\left(l-u_{g, i}\right)^{2}}{2 \tilde{\sigma}_{g, i}^{2}}\right] \hat{Q}_{i}(l) G_{i}\left(u_{g, i}\right) .
\end{aligned}
$$

With $\hat{f}_{\boldsymbol{L}, i}\left(u_{g, i}\right)=\hat{Q}_{i}\left(u_{g, i}\right) G_{i}\left(u_{g, i}\right)$, and replacing $\hat{f}_{\boldsymbol{L}, i}\left(u_{g, i}\right)$ by $f_{\boldsymbol{L}, i}\left(u_{g, i}\right)$, (48) can be rewritten as

$$
\hat{f}_{\boldsymbol{L}, i}(l)=\hat{Q}_{i}(l)\left\{\begin{array}{l}
\sqrt{2 \tilde{\sigma}_{g, i}^{2}} D_{+}\left(\frac{l-u_{g, i}}{\sqrt{2 \tilde{\sigma}_{g, i}^{2}}}\right) \\
+\exp \left[-\frac{\left(l-u_{g, i}^{2}\right)^{2}}{2 \tilde{\sigma}_{g, i}^{2}}\right] \frac{f_{\boldsymbol{L}, i}\left(u_{g, i}\right)}{\hat{Q}_{i}\left(u_{g, i}\right)}
\end{array}\right\} .
$$

Substituting (49) into (29), the PDF of path losses consisting of the propagation attenuation, shadowing and antenna loss can be approximated as

$$
\hat{f}_{\boldsymbol{L}}(l)=\sum_{i=1}^{M} \lambda_{i} \hat{Q}_{i}(l)\left\{\begin{array}{l}
\sqrt{2 \tilde{\sigma}_{g, i}^{2}} D_{+}\left(\frac{l-u_{g, i}}{\sqrt{2 \tilde{\sigma}_{g, i}^{2}}}\right) \\
+\exp \left[-\frac{\left(l-u_{g, i}\right)^{2}}{2 \tilde{\sigma}_{g, i}^{2}}\right] \frac{f_{L, i}\left(u_{g, i}\right)}{\hat{Q}_{i}\left(u_{g, i}\right)}
\end{array}\right\} .
$$

With the PDF of path losses in close form, the outage probability [6] that path losses are greater than the outage threshold $l_{\text {out }}$ can be computed by

$$
P_{\text {out }}=\int_{l_{\text {out }}}^{+\infty} \hat{f}_{\boldsymbol{L}}(l) d l .
$$

\section{Error Analysis}

Without loss of generality, assuming $l \geq l_{0}$, the relative error between $\hat{f}_{\boldsymbol{L}, i}(l)$ and $f_{\boldsymbol{L}, i}$ can be expressed as

$$
\begin{aligned}
& \left|\frac{\hat{f}_{\boldsymbol{L}, i}(l)-f_{\boldsymbol{L}, i}(l)}{f_{\boldsymbol{L}, i}(l)}\right| \\
& =\left|\frac{\int_{l_{0}}^{l}\left(\hat{Q}_{i}(t)-Q_{i}(t)\right) \exp \left(\int_{l_{0}}^{t} P_{i}(s) d s\right) d t}{\int_{l_{0}}^{l} Q_{i}(t) \exp \left(\int_{l_{0}}^{t} P_{i}(s) d s\right) d t+f_{\boldsymbol{L}, i}\left(l_{0}\right)}\right| \\
& =\frac{\left|\int_{l_{0}}^{l} \frac{\hat{Q}(t)-Q_{i}(t)}{Q_{i}(t)} Q_{i}(t) \exp \left(\int_{l_{0}}^{t} P_{i}(s) d s\right) d t\right|}{\int_{l_{0}}^{l} Q_{i}(t) \exp \left(\int_{l_{0}}^{t} P_{i}(s) d s\right) d t+f_{\boldsymbol{L}, i}\left(l_{0}\right)}
\end{aligned}
$$




$$
\begin{aligned}
& \leq \sup _{l_{0} \leq t \leq l}\left|\frac{\hat{Q}_{i}(t)-Q_{i}(t)}{Q_{i}(t)}\right| \int_{l_{0}}^{l} Q_{i}(t) \exp \left(\int_{l_{0}}^{t} P_{i}(s) d s\right) d t \\
& \int_{l_{0}}^{l} Q_{i}(t) \exp \left(\int_{l_{0}}^{t} P_{i}(s) d s\right) d t+f_{\boldsymbol{L}, i}\left(l_{0}\right) \\
& \leq \sup _{l_{0} \leq t \leq l}\left|\frac{\hat{Q}_{i}(t)-Q_{i}(t)}{Q_{i}(t)}\right| .
\end{aligned}
$$

We can see that the relative error of the approximation to the PDF of path losses is upper bounded by the least upper bound of that of the Gaussian approximation to $Q_{i}(l)$ in the differential equation. Therefore the error propagation resulting from the Gaussian approximation to the exciting source of the differential equation can be ignored.

\section{Analytical and Simulation Results}

In this section, the performances of the GMM, Gaussian approximation to the exciting source of the differential equation, and approximate PDF of path losses $\boldsymbol{L}$ are investigated for sectorized cellular networks [1] [26] where UEs are uniformly distributed. Numerical computation and Monte Carlo simulation are performed to verify the derivation and show the impacts of the order of the GMM, attenuation exponent and standard deviation of the shadowing. The main simulation parameters are presented in Table III.

TABLE III

SIMULATION PARAMETERS.

\begin{tabular}{cc}
\hline System parameter & Parameter value \\
\hline Cell radius & $100 \mathrm{~m}$ \\
Reference distance & $1 \mathrm{~m}$ \\
Path loss at the reference distance & $37 \mathrm{~dB}$ \\
Maximum antenna gain plus cable loss & $14 \mathrm{dBi}$ \\
Number of sectors in a cell & 3 \\
3dB beam-width in degrees & $70^{\circ}$ \\
Maximum antenna attenuation & $20 \mathrm{~dB}$ \\
Attenuation exponent & $2 \sim 4$ \\
Standard deviation of the SF & $3 \sim 12 \mathrm{~dB}$ \\
\hline
\end{tabular}

\section{A. PDF of Propagation Attenuation plus Shadowing}

In this sub-section, the PDF of the addition of the propagation attenuation and shadowing $\boldsymbol{Y}$ and performance of the GMM are investigated in terms of ASR. To investigate the impact of ASR on the shape of the PDF, we define the normalized random variable $\overline{\boldsymbol{Y}}=\frac{\boldsymbol{Y}-c_{\boldsymbol{Y}}^{1}}{\sqrt{c_{\boldsymbol{Y}}^{2}}}$ where $c_{\boldsymbol{Y}}^{1}$ and $c_{\boldsymbol{Y}}^{2}$ are the first two cumulants of $\boldsymbol{Y}$. As can be seen from Fig. 3, if the different values of the attenuation exponent and standard deviation of the shadowing have the same ASR, the shapes of distributions are identical. From Fig. 4, we can see that the skewness of $\overline{\boldsymbol{Y}}$ will tend to zero with the decrease of the ASR. Only when the ASR is small, the PDF of $\overline{\boldsymbol{Y}}$ can be well approximated as the standard Gaussian distribution. In other words, it is not feasible to assume that the addition of the propagation attenuation and shadowing is normally distributed when ASR is large.

\section{B. Differential Equation with Approximated Exciting Source}

To make the convolution of the Gaussian function $N\left(l ; \tilde{u}_{i}, \tilde{\sigma}_{i}^{2}\right)$ and antenna loss $f_{\boldsymbol{Z}}(z)$ tractable, the exciting

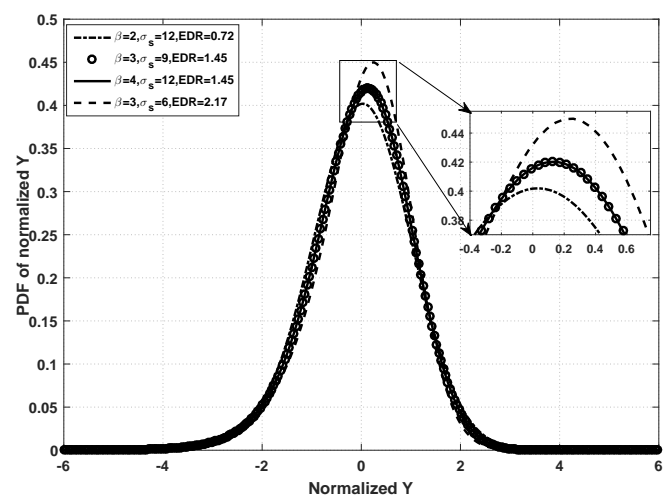

Fig. 3. PDFs of $\overline{\boldsymbol{Y}}$ in terms of the metric ASR.

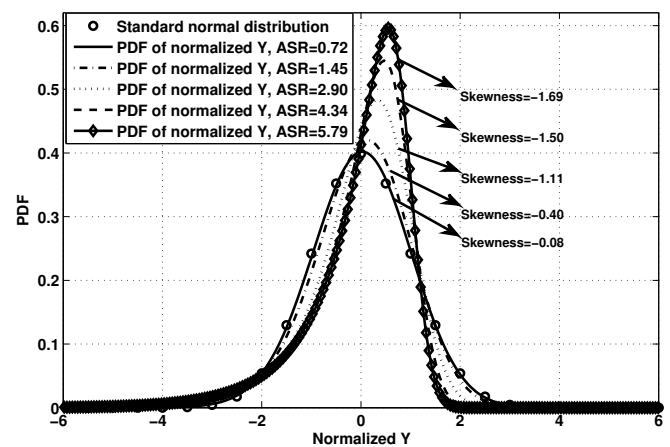

Fig. 4. Standard normal distribution and PDFs of $\overline{\boldsymbol{Y}}$.

source of the differential equation (31) which is transformed from the convolution operation is approximated as the Gaussian function $\hat{Q}_{i}(l)$. In this sub-section, the performance of the Gaussian approximation $\hat{Q}_{i}(l)$ is investigated. We use the $3 \mathrm{~dB}$ beam-width of the antenna pattern $\theta_{k}=\frac{7}{6} \frac{\pi}{k}$ as specified in [1] [27]. To utilize the KL divergence to evaluate the performance of the Gaussian approximation, the function $\hat{Q}_{i}(l)$ and $Q_{i}(l)$ are normalized to be PDFs. The KL

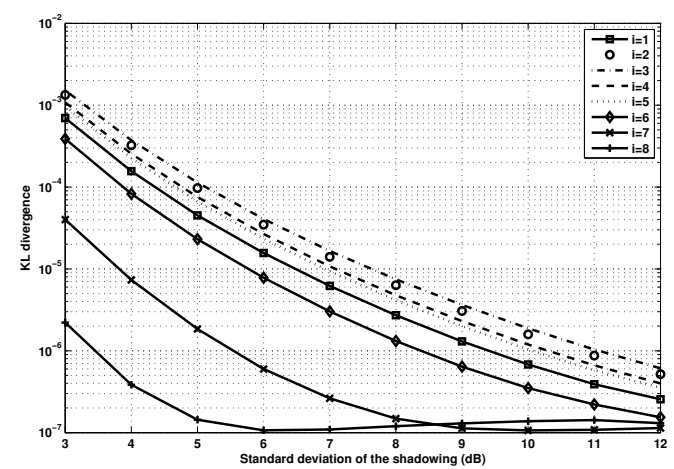

Fig. 5. KL divergence of the normalized $\hat{Q}_{i}(l)$ from the normalized $Q_{i}(l)$ versus the standard deviation of the shadowing when the order of the GMM is eight.

divergence of the normalized $\hat{Q}_{i}(l)$ from the normalized $Q_{i}(l)$ is shown in Fig. 5 when the order of the Gaussian mixture 
model is eight. It will quickly approach zero with the increase of the standard deviation of the shadowing. Therefore the exciting source $Q_{i}(l)$ can be well approximated as a weighted Gaussian function as given in (39).

\section{PDF of Path Loss}

In this sub-section, the approximate PDF of the addition of the propagation attenuation, shadowing and antenna loss $\boldsymbol{L}$ represented by Gaussian functions and Dawson functions is investigated in terms of the attenuation exponent and standard deviation of the shadowing. The PDF of $\boldsymbol{L}$ is obtained by the numerical computation.

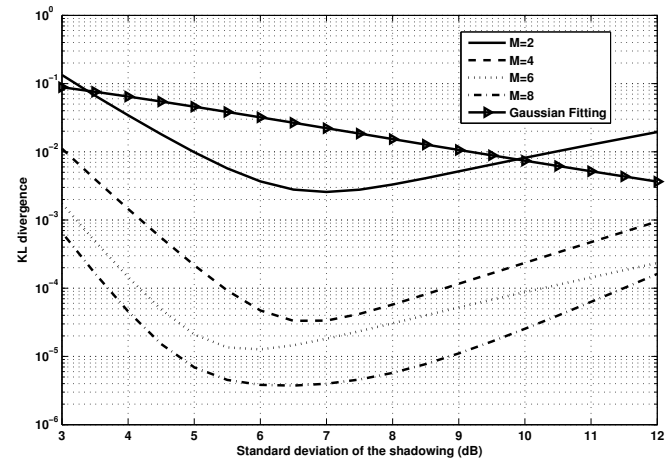

Fig. 6. KL divergence of $\hat{f}_{\boldsymbol{L}}(l)$ and Gaussian fitting from $f_{\boldsymbol{L}}(l)$ versus the standard deviation of the shadowing when the attenuation exponent is three.

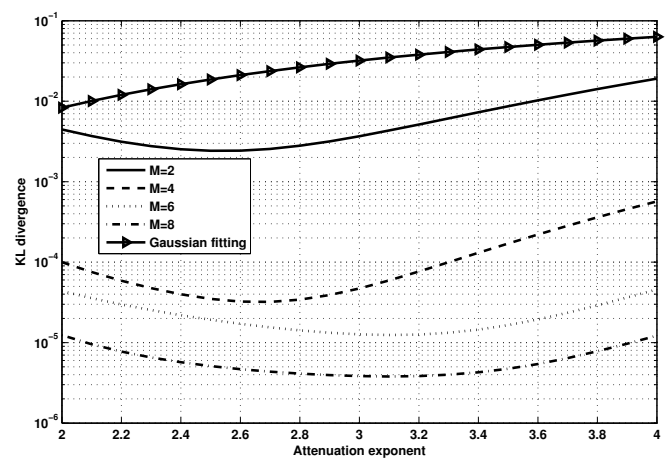

Fig. 7. KL divergence of $\hat{f}_{\boldsymbol{L}}(l)$ and Gaussian fitting from $f_{\boldsymbol{L}}(l)$ versus the attenuation exponent when the standard deviation of the shadowing is $6 \mathrm{~dB}$.

The KL divergence of the approximate PDF $\hat{f}_{\boldsymbol{L}}(l)$ from $f_{\boldsymbol{L}}(l)$ is shown in Fig. 6 and Fig. 7 in terms of the standard deviation of the shadowing and attenuation exponent, respectively. The KL divergence of the Gaussian fitting with the same mean and variance as $f_{\boldsymbol{L}}(l)$ from $f_{\boldsymbol{L}}(l)$ is also compared. Results show that the PDF of path losses will approach a Gaussian function when the standard deviation of the shadowing increases or the attenuation exponent decreases. If we assume that the PDF of path losses can be considered Gaussian when the KL divergence is smaller than 0.01, the PDF of path losses cannot be approximated as a Gaussian function when the standard deviation of the shadowing is less than $9.2 \mathrm{~dB}$. When the order of Gaussian mixture model is not less than four, the PDF of path losses can be tightly approximated in the form of Dawson functions and Gaussian functions.

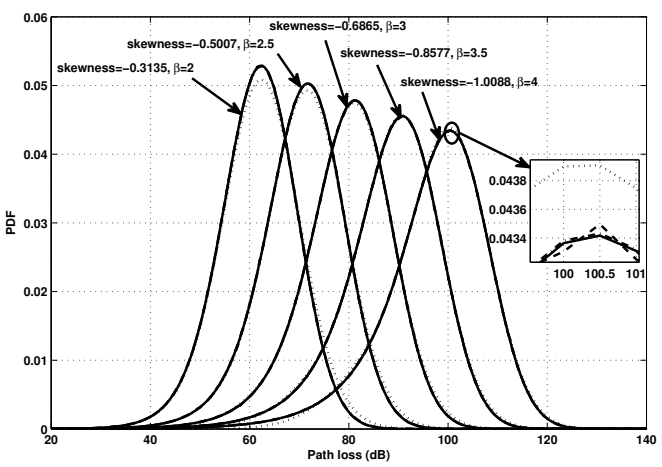

Fig. 8. PDFs of $f_{\boldsymbol{L}}(l)$ and $\hat{f}_{\boldsymbol{L}}(l)$ when the standard deviation of the shadowing is $6 \mathrm{~dB}$. The solid lines are the exact PDFs through numerical computation. The dashed lines are the Monte Carlo simulation results. The dotted lines are the approximations of the PDFs when the order of the Gaussian mixture model is two. The dash-dotted lies are the approximations of the PDFs when the order of the Gaussian mixture model is four.

The PDF curves in Fig. 8 show that the PDF of path losses deviates from the Gaussian function with the increase of the attenuation exponent. The increase of the attenuation exponent will destroy the symmetry of the PDF of path losses and the tail on the left side of the PDF of path losses will become longer than the right side. When the order of the Gaussian mixture model is not less than four, the derived closed-form PDF of path losses always achieves satisfactory performance.

Fig. 9 shows that the outage probability based on the Gaussian fitting is not accurate when the cell radius is small or the outage threshold is large. And the cell radius will decrease with the increase of the standard deviation if the target outage probability is given. Outage probabilities based on $\hat{f}_{\boldsymbol{L}}(l)$ are obtained by the numerical computation. The performance gap between outage probabilities based on $\hat{f}_{\boldsymbol{L}}(l)$ and Monte Carlo simulation can be ignored. If the desired outage probability for the network planning is less than $2 \%$, the cell size computed through the Gaussian fitting will be significantly smaller than that computed through $\hat{f}_{\boldsymbol{L}}(l)$ and Monte Carlo simulation. Therefore, the cell size computed through the Gaussian fitting will result in more BSs than needed.

\section{CONCLUSIONS}

In this paper, the PDF of path losses including the propagation attenuation, shadowing and antenna pattern is studied, and a closed-form approximation of the PDF of path losses is derived. The cumulant analysis shows that the defined metric ASR determines the shape of the PDF of the addition of the propagation attenuation and shadowing. Only when the ASR is small, the assumption that the addition of the propagation attenuation and shadowing is normally distributed is feasible. Otherwise, it greatly deviates from the normal distribution. Numerical and Monte Carlo simulation results show that the closed-form approximation can always achieve the satisfactory performance. Moreover, the outage probability 


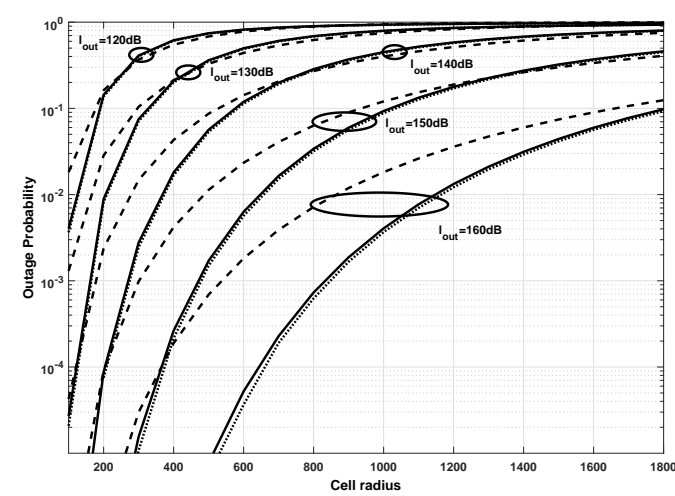

(a) Standard deviation of shadowing is $6 \mathrm{~dB}$.

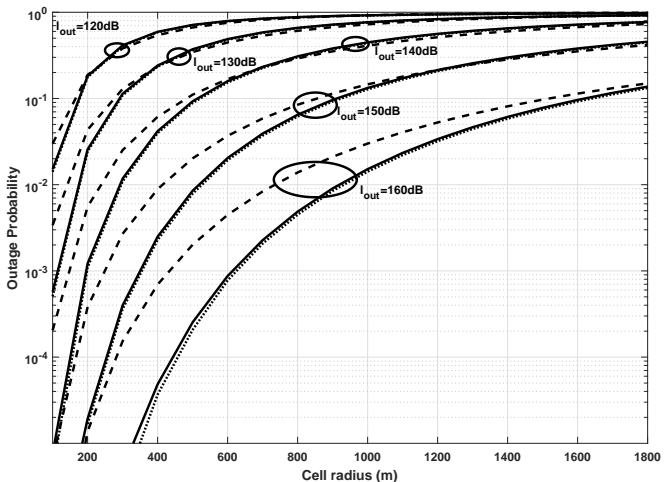

(b) Standard deviation of shadowing is $8 \mathrm{~dB}$.

Fig. 9. Outage probabilities based on $\hat{f}_{\boldsymbol{L}}(l)$ and Monte Carlo simulation when the attenuation exponent is four. The dotted, dashed and solid lines denote the outage probability based on the Monte Carlo simulation, Gaussian approximation and $\hat{f}_{\boldsymbol{L}}(l)$ respectively.

computed through the derived closed-form approximation is more accurate than that computed through the Gaussian approximation. With a given outage threshold, the cell size can be better designed through the closed-form PDF of path losses than through the Gaussian fitting.

\section{APPENDIX A \\ INDEPENDENCE OF $\boldsymbol{D}$ AND $\boldsymbol{\Theta}$}

Without loss of generality, the proof is given for the threesector cell site. With the assumption that UEs are uniformly distributed in the three-sector cell site, the probability that UEs locate in the colored area of Fig. 10 is

$$
\begin{aligned}
F_{\boldsymbol{\Theta}, \boldsymbol{D}}(\theta, d) & =P(\boldsymbol{\Theta} \leq \theta, \boldsymbol{D} \leq d) \\
& =\int_{0}^{\theta} \int_{0}^{d} f_{\boldsymbol{\Theta}, \boldsymbol{D}}(x, y) d x d y=\frac{\theta d^{2}}{\frac{2 \pi}{3} R^{2}} .
\end{aligned}
$$

The joint PDF of the random variable $\Theta$ and $D$ is

$$
f_{\boldsymbol{\Theta}, \boldsymbol{D}}(\theta, d)=\frac{\partial^{2} F_{\boldsymbol{\Theta}, \boldsymbol{D}}(\theta, d)}{\partial \theta \partial d}=\frac{1}{\frac{2 \pi}{3}} \frac{2 d}{R^{2}} .
$$

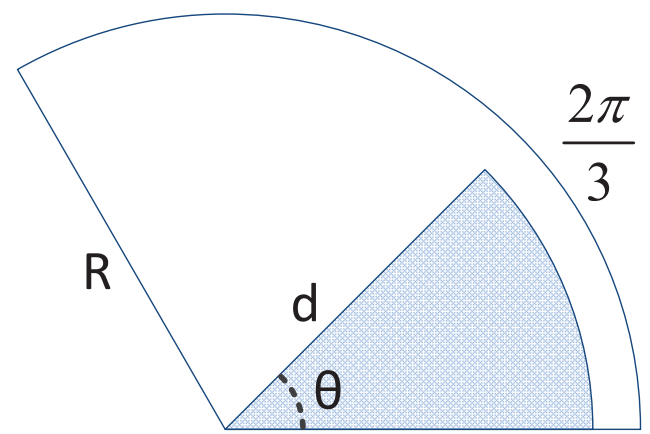

Fig. 10. Illustration of the UE distribution.

The marginal PDFs of the random variable $\Theta$ and $D$ can be derived from the joint PDF in (54) as

$$
f_{\boldsymbol{\Theta}}(\theta)=\frac{1}{\frac{2 \pi}{3}}, f_{\boldsymbol{D}}(d)=\frac{2 d}{R^{2}}
$$

Therefore

$$
f_{\boldsymbol{\Theta}, \boldsymbol{D}}(\theta, d)=f_{\boldsymbol{\Theta}}(\theta) f_{\boldsymbol{D}}(d)
$$

\section{APPENDIX B}

General Solution of DiffEREnTIAL EQuATION

Assume that

$$
f_{\boldsymbol{L}, i}(l)=u_{i}(l) v_{i}(l)
$$

Substituting (57) into (31), we have

$$
u_{i}^{\prime}(l) v_{i}(l)+u_{i}(l)\left(v_{i}^{\prime}(l)+P_{i}(l) v_{i}(l)\right)=Q_{i}(l) .
$$

Assume that

$$
v_{i}^{\prime}(l)+P_{i}(l) v_{i}(l)=0 .
$$

Then the function $v_{i}(l)$ can be expressed as

$$
v_{i}(l)=v_{i}\left(l_{0}\right) \exp \left(-\int_{l_{0}}^{l} P_{i}(s) d s\right) .
$$

Substituting (60) into (58), we obtain

$$
u_{i}(l)=\frac{1}{v_{i}\left(l_{0}\right)} \int_{l_{0}}^{l} Q_{i}(t) \exp \left(\int_{l_{0}}^{t} P_{i}(s) d s\right)+u_{i}\left(l_{0}\right) .
$$

Combining (61) and (60), we have

$$
\begin{aligned}
f_{\boldsymbol{L}, i}(l)= & \exp \left(-\int_{l_{0}}^{l} P_{i}(s) d s\right) \\
& \times\left[\int_{l_{0}}^{l} Q_{i}(t) \exp \left(\int_{l_{0}}^{t} P_{i}(s) d s\right) d t+f_{\boldsymbol{L}, i}\left(l_{0}\right)\right] .
\end{aligned}
$$




\section{REFERENCES}

[1] 3GPP TR 36.814, "Evolved universal terrestrial radio access (EUTRA);further advancements for E-UTRA physical layer aspects,' v9.0.0, Mar. 2010.

[2] P. Kabir, M. Shafinia, and F. Marvasti, "Capacity bounds of finite dimensional CDMA systems with power allocation, fading, and nearfar effects," IEEE Commun. Lett., vol. 17, no. 1, pp. 15-18, Jan. 2013.

[3] U. Mitra and H. V. Poor, "Adaptive receiver algorithms for near-far resistant CDMA," in Proc. IEEE PIMRC, Oct. 1992, pp. 639-644.

[4] S. Chen and J. Zhao, "The requirements, challenges, and technologies for $5 \mathrm{G}$ of terrestrial mobile telecommunication," IEEE Commun. Mag., vol. 52, no. 5, pp. 36-43, May 2014.

[5] C.-X. Wang, F. Haider, X. Gao, X.-H. You, Y. Yang, D. Yuan, H. Aggoune, H. Haas, S. Fletcher, and E. Hepsaydir, "Cellular architecture and key technologies for $5 \mathrm{G}$ wireless communication networks," IEEE Commun. Mag., vol. 52, no. 2, pp. 122-130, Feb. 2014.

[6] M. J. Feuerstein, K. L. Blackard, T. S. Rappaport, S. Y. Seidel, and H. Xia, "Path loss, delay spread, and outage models as functions of antenna height for microcellular system design," IEEE Trans. Veh. Technol., vol. 43, no. 3, pp. 487-498, Aug. 1994.

[7] X. Ge, B. Yang, J. Ye, G. Mao, C. X. Wang, and T. Han, "Spatial spectrum and energy efficiency of random cellular networks," IEEE Trans. Commun., vol. 63, no. 3, pp. 1019-1030, Mar. 2015.

[8] X. Yan, J. Xu, Y. Zhu, J. Wang, Y. Yang, and C. X. Wang, "Downlink average rate and SINR distribution in cellular networks," IEEE Trans. Commun., vol. 64, no. 2, pp. 847-862, Feb. 2016.

[9] C. U. Castellanos, D. L. Villa, C. Rosa, K. I. Pedersen, F. D. Calabrese, P.-H. Michaelsen, and J. Michel, "Performance of uplink fractional power control in utran LTE," in Proc. IEEE VTC, May 2008, pp. 25172521.

[10] R. Madan and S. Ray, "Uplink resource allocation for frequency selective channels and fractional power control in LTE," in Proc. IEEE ICC, Jun. 2011, pp. 1-5.

[11] K. Majewski and M. Koonert, "Analytic uplink cell load approximation for planning fractional power control in LTE networks," in Proc. IEEE NETWORKS, Sep. 2010, pp. 1-7.

[12] H. Zhang, N. Prasad, S. Rangarajan, S. Mekhail, S. Said, and R. Arnott, "Standards-compliant LTE and LTE-A uplink power control," in Proc. IEEE ICC, Jun. 2012, pp. 10-15.

[13] Z. Bharucha and H. Haas, "The distribution of path losses for uniformly distributed nodes in a circle," Research Lett. Commun., vol. 2008, no. 4, p. 4 pages, Jan. 2008 .

[14] Y. Broyde and H. Messer, "A cellular sector-to-users path loss distribution model," in Proc. IEEE/SP SSP, Aug. 2009, pp. 312-324.

[15] T. M. Cover and J. A. Thomas, Elements of Information Theory. John Wiley, 1991.

[16] S. Baroudi and Y. R. Shayan, "Outage probability in a circle with uniformly distributed users," in Proc. IEEE CCECE, Apr. 2012, pp. $1-4$.

[17] M. Abdulla and Y. R. Shayan, "Closed-form path-loss predictor for Gaussianly distributed nodes," in Proc. IEEE ICC, May 2010, pp. 1-6.

[18] K. B. Baltzis, "Analytical and closed-form expressions for the distribution of path loss in hexagonal cellular networks," Wireless Pers. Commun., vol. 60, no. 4, pp. 599-610, Oct. 2011.

[19] M. Abdulla and Y. R. Shayan, "An exact path-loss density model for mobiles in a cellular system," in Proc, ACM MMWA, Oct. 2009, pp. $118-122$.

[20] K. B. Baltzis, "Closed-form description of microwave signal attenuation in cellular systems," Radio Eng., vol. 19, no. 1, pp. 11-16, Apr. 2010.

[21] K. Govindan, K. Zeng, and P. Mohapatra, "Probability density of the received power in mobile networks," IEEE Trans. Wireless Commun., vol. 10, no. 11, pp. 3613-3619, Nov. 2011.

[22] W. H. Press, B. P. Flannery, S. A. Teukolsky, and W. T. Vetterling, Numerical Recipes: The Art of Scientific Computing, 3rd ed. Cambridge university press, 2007.

[23] M. H. Islam, C. L. Koh, S. W. Oh, X. Qing, Y. Y. Lai, C. Wang, Y.-C. Liang, B. E. Toh, F. Chin, G. L. Tan et al., "Spectrum survey in singapore: Occupancy measurements and analyses," in Proc. IEEE CROWNCOM, May 2008, pp. 1-7.

[24] T. Zhou, M. Peng, W. Wang, and H.-H. Chen, "Low-complexity coordinated beamforming for downlink multicell SDMA/OFDM systems," IEEE Trans. Veh. Technol., vol. 62, no. 1, pp. 247-255, Jan. 2013.

[25] X. Ge, K. Huang, C.-X. Wang, X. Hong, and X. Yang, "Capacity analysis of a multi-cell multi-antenna cooperative cellular network with co-channel interference," IEEE Trans. Wireless Commun., vol. 10, no. 10 , pp. 3298-3309, Oct. 2011.
[26] ETSI TR 125 996, "Universal mobile telecommunications system (UMT$\mathrm{S}$ ); spacial channel model for multiple input multiple output (MIMO) simulations," v9.0.0, Jan. 2010.

[27] IEEE 802.16 Broadband Wireless Access Working Group, "IEEE 802.16m evaluation methodology document," Jan. 2009.

[28] S. R. Saunders, Antennas and Propagation for Wireless Communication Systems. John Wiley \& Sons, 2008.

[29] A. Hyvärinen, J. Karhunen, and E. Oja, Independent Component Analysis. John Wiley \& Sons, 2004.

[30] T. Steihaug, "The conjugate gradient method and trust regions in large scale optimization," Siam Journal on Numerical Analysis, vol. 20, no. 3, pp. 626-637, 1983.

[31] R. H. Byrd, R. B. Schnabel, and G. A. Shultz, "Approximate solution of the trust region problem by minimization over two-dimensional subspaces," Mathematical Programming, vol. 40, no. 1, pp. 247-263, 1988. 